\title{
Serological characterization of the core region of lipopolysaccharides of rough Proteus sp. strains
}

\section{Agata Palusiak and Zygmunt Sidorczyk}

Department of General Microbiology, Institute of Microbiology, Biotechnology and Immunology, University of Łódź, Poland

Received: 2008.02.22, Accepted: 2008.10.29, Published online: 2009.07.04

(C) L. Hirszfeld Institute of Immunology and Experimental Therapy, Wrocław, Poland 2009

\begin{abstract}
Introduction: Both smooth and rough Proteus sp. strains can be found. The latter are characterized by their lack of an O-polysaccharide chain in the lipopolysaccharide (LPS) molecule, which makes them suitable for obtaining anti-core sera. Using this kind of material enables identifying fragments of the Proteus LPS core region that might be involved in cross-reactions. To date only a few similar epitopes have been established for the genus Proteus.

Materials and Methods: Polyclonal rabbit antisera directed against three rough strains of Proteus sp. were tested by enzymelinked immunosorbent assay (ELISA) with a set of LPSs. The reactivity of the selected cross-reactive and homologous systems was checked by the Western blot technique and by a passive immunohemolysis assay preceded by the absorption of each antiserum with appropriate cross-reactive and homologous alkalized LPSs.

Results: On the basis of the ELISA results, 19 cross-reactive antigens were selected among which both smooth and rough LPS forms were found. All the observed reactions involved the core region of the LPS. Using the antisera absorbed with the appropriate LPSs allowed identification of four groups of antigens with serologically identical core regions.

Conclusions: Comparing the results of the serological studies with the known chemical structures of the core regions of the LPSs used enabled the identification of a few core oligosaccharide fragments probably involved in the observed cross-reactions. All were located in the most distal part of LPS core region, which made them more easily recognized by specific antibodies.
\end{abstract}

Key words: Proteus, lipopolysaccharide, core region, rough strains.

Abbreviations: ELISA - enzyme-linked immunosorbent assay, GalA - galacturonic acid, GalN - galactosamine, Glc - glucose, GlcN - glucosamine, Hep - L-glycero-D-manno-heptose, DD-Hep - D-glycero-D-manno-heptose, PIH - passive immunohemolysis, SDS/PAGE - sodium dodecyl sulfate polyacrylamide gel electrophoresis, SRBC - sheep red blood cells, $(1 S)$-GaloNAc - open-chain form of GalNAc.

Corresponding author: Agata Palusiak, Department of General Microbiology, Institute of Microbiology, Biotechnology and Immunology, University of Lódź, Banacha 12/16, 90-237 Łódź, Poland, tel.: +48 42-6354469, fax: +48 42-6655818, e-mail: agatapal@biol.uni.lodz.pl

\section{INTRODUCTION}

Lipopolysaccharide (LPS) is considered one of the most important virulence factors of Gram-negative bacilli of the genus Proteus. It comprises three regions differing in chemical structure and biological properties: lipid A, an O-polysaccharide chain, and an intervening core region (Vinogradov et al. 2002b). Bacteria which possess all parts of LPS are called smooth (S) forms and those lacking the outer-most part, i.e. the O-polysaccharide chain, are referred to as rough (R) ones (Poxton 1995). The latter strains can be isolated from clinical materials (e.g. P. penneri 0541-74) (Hickman et al. 1982) or obtained in the laboratory, like the mutant R110 iso- lated after induction performed on a wild strain $(P$. mirabilis S1959) by methyl p-toluenesulphonate (Kotełko et al. 1977). In antisera obtained against rough strains, no O-polysaccharide-specific antibodies can be found, which is why such antisera are ideal material for determining the serological specificity of the Proteus sp. LPS core region. This is very important because knowledge in this matter is quite confined. To date only a few structural elements have been determined in Proteus LPS core regions as involved in cross-reactions. The first one, D-GalpA6(L-Lys), was present in the core region of $P$. mirabilis $\mathrm{O} 28$ LPS and the other, $\beta$-D-Quip $4 \mathrm{~N}(\mathrm{~L}-$ Ala), was found in the $P$. vulgaris TG 103 (O54) and $P$. mirabilis O6 core oligosaccharides (Kołodziejska et al. 
2006; Sidorczyk et al. 2002c). Both fragments were identified in the LPS core regions of smooth Proteus sp. strains. The present study concerns determining the epitope specificities of the core regions of rough strains. Although these strains are not isolated from patients as often as smooth ones, it is still important to examine the serological specificity of their LPS core regions and the nature of the cross-reactions in which they are involved. Such knowledge may prove helpful in the future when choosing appropriate $\mathrm{R}$ antigens to prepare a vaccine against infections caused by both smooth and rough Proteus sp. strains.

\section{MATERIALS AND METHODS}

\section{Bacterial cells and cultivation}

R110 mutant was isolated at the Department of General Microbiology, University of Łódź, Poland, after induction performed on the wild strain $P$. mirabilis S1959. P. penneri 13, 37, and 44 ( $\mathrm{R}$ forms) were clinical isolates from patients from New Jersey (P. penneri 13) and Toronto (P. penneri 37 and 44). These strains and 24 others, i.e. P. penneri 1 (O72a), 2 (O66), 4 (O72a,b), 7 (O61), 8 (O67), 11 (O58), 12 (O58), 14 (O59), 15 (O52), 16 (O17), 17 (O8), 18 (O17), 19 (O64a,b,c), 22 (O63), 25 (O69), 26 (O31a), 27 (O64a,b,c), 28 (O31a,b), 31 (O19a,b), 34 (O65), 39, 40 (O64a,b,d), 41 (O62), and 42 (O71), were kindly provided by Prof. D. J. Brenner, Centers for Disease Control and Prevention in Atlanta, GA, USA. P. penneri 60 (O70), 63 (O68), and 75 $(\mathrm{O} 73 \mathrm{a}, \mathrm{c})$ were isolated from the urine of patients with bacteriuria in a hospital in Łódź and, like $P$. penneri 71 $(\mathrm{O} 64 \mathrm{a}, \mathrm{c}, \mathrm{e})$, they belong to the collection of the Department of General Microbiology, University of Łódź. The other strains were $P$. penneri 47 (O59), kindly provided by Dr. E. Falsen, Department of Clinical Bacteriology, Göteborg, Sweden; P. penneri 77 (O65) and 93 (O67) by Dr. B. Senior, Department of Medical Microbiology in Dundee, UK; P. penneri 103 (O73a,b), 104 (O61), 107 (O8), 112 (O8), and P. penneri 124 (R form) by Dr. B. Holmes, Central Public Health Laboratory in London, UK; and P. penneri 133 (O61) by Dr. G. Giammanco, Institute of Hygiene and Prevention Medicine in Catania, Italy. The bacteria were grown under aerobic conditions in liquid nutrient broth containing $0.1 \%$ glucose (BTL, Poland) for $18 \mathrm{~h}$ at $37^{\circ} \mathrm{C}$, killed with phenol, centrifuged, washed twice with water, and lyophilized to obtain dried bacterial cells.

\section{Isolation and alkalization of LPSS}

LPSs were isolated from the bacterial $\mathrm{R}$ forms by the phenol/chloroform/petroleum ether method of Galanos (Galanos et al. 1969). Alkali-treated LPSs were obtained by saponification of the crude LPS preparations with $0.25 \mathrm{M} \mathrm{NaOH}\left(2 \mathrm{~h}, 56^{\circ} \mathrm{C}\right)$ and precipitation with $96 \%$ ethanol (Kołodziejska et al. 2006).
Immunization procedures and sources of the sera

Rabbit polyclonal antisera against whole cells of $P$. penneri 37 and 44 and mutant R110 were obtained by intravenous immunization of New Zealand white rabbits with $0.25,0.5$, and $1.0 \mathrm{ml}$ of heat-inactivated bacteria suspended in $0.85 \% \mathrm{NaCl}\left(1.5 \times 10^{10} \mathrm{cfu} / \mathrm{ml}\right)$ at days 0 , 5 , and 10. The animals were bled one week after the last immunization (Sidorczyk et al. 2002c). Serum against whole cells of $P$. penneri 13 was provided by the Department of General Microbiology, University of Łódź.

\section{Serological assays}

Passive immunohemolysis (PIH), sodium dodecyl sulfate polyacrylamide gel electrophoresis (SDS/ /PAGE), Western blot, and enzyme-linked immunosorbent assay (ELISA) were carried out as previously described (Sidorczyk et al. 2002c). For PIH, sheep red blood cells (SRBCs) were sensitized with alkali-treated LPSs (64-200 $\mu \mathrm{g} / 0.2 \mathrm{ml}$ of SRBCs). For ELISA, polystyrene microtiter plates were coated with 50-200 ng LPS per well. Antibody titer determination (the highest dilution of antiserum giving optical density $405 \geq 0.2$ ) was repeated three times for each system. For Western blot the antisera were diluted 1:100 in dot-blot-10\% skimmed milk buffer.

\section{Absorption procedure}

The antiserum was diluted 1:50 with veronal buffer, $\mathrm{pH} 7.3$, and incubated with SRBCs $(0.2 \mathrm{ml})$ sensitized with the respective alkali-treated LPSs $(200 \mu \mathrm{g})$ of one Proteus strain for $30 \mathrm{~min}$ in ice. Antibodies that bound to the LPS used for the absorption were removed from the antiserum by centrifugation. The reactivities of twofold serially diluted antisera were tested by PIH. The antiserum titer was established as the last dilution of antiserum resulting in 50\% hemolysis (Sidorczyk et al. 2002c).

\section{RESULTS AND DISCUSSION}

Three polyclonal rabbit antisera directed against the rough strains of the genus Proteus (P. penneri 13 and 37 and $P$. mirabilis R110/S1959) were checked by ELISA with the set of 41 Proteus LPSs used as antigens and selected for this study on the basis of the results of previous serological studies conducted at the Department of General Microbiology (Drzewiecka et al. 2004; Kondakova et al. 2003; Sidorczyk et al. 2002a; Sidorczyk et al. 2002b; Zych et al. 2000; Zych et al. 2005). The technique was applied with the aim of finding the crossreactive systems and showing the strength of their reactions.

As can be seen in Table 1, P. penneri 13 antiserum cross-reacted with eight antigens, of which four LPSs $(P$. 
Table 1. Reactivity of antisera against $P$. penneri 13,37 , and P. mirabilis R110 with the Proteus sp. LPSs ${ }^{\mathrm{a}}$

\begin{tabular}{cc}
\hline LPS from Proteus sp. strains & $\begin{array}{c}\text { Reciprocal titer } \\
\text { for the LPS in ELISA }\end{array}$ \\
\hline P. penneri 13 antiserum & \\
P. penneri 13 & 16,000 \\
P. penneri 112 & 16,000 \\
P. penneri 12 & 16,000 \\
P. penneri 124 & 16,000 \\
P. penneri 26 & 16,000 \\
P. penneri 17 & 8,000 \\
P. penneri 7 & 4,000 \\
P. penneri 37 & 4,000 \\
P. penneri 77 & 4,000 \\
P. penneri 37 antiserum & \\
P. penneri 37 & 32,000 \\
P. penneri 44 & 32,000 \\
P. penneri 77 & 16,000 \\
P. penneri 7 & 2,000 \\
P. penneri 13 & 4,000 \\
P. penneri 17 & 4,000 \\
P. penneri 26 & 4,000 \\
P. penneri 112 & 4,000 \\
P. mirabilis $\mathrm{R} 110$ antiserum & \\
P. mirabilis $\mathrm{R} 110$ & 32,000 \\
P. penneri 7 & 4,000 \\
P. penneri 14 & 4,000 \\
P. penneri 15 & 8,000 \\
P. penneri 47 & 16,000 \\
\hline
\end{tabular}

${ }^{\text {a }}$ Data for the homologous LPSs are italicized.

penneri $12,26,112$, and 124$)$ bound antibodies in a manner identical to the homologous antigen, one (P. penneri $17)$ reacted slightly more weakly, and the titers obtained for the last three (P. penneri 7, 37, and 77) were only 1:4000. The second antiserum, directed against the $P$. penneri 37 strain, cross-reacted with seven LPS preparations, with $P$. penneri 44 reacting in the same way as the homologous antigen, $P$. penneri 77 binding immunoglobulins similar to the first two antigens mentioned, and the last five LPSs (P. penneri 7, 13, 17, 26, and 112) reacting with the lowest intensity. The third antiserum, against mutant R110, cross-reacted with the lowest number of antigens, of which only one (P. penneri 47) bound antibodies nearly as strongly as the tested antigen while the remaining LPS preparations ( $P$. penneri 7,14 , and 15 ) reacted more weakly.

To examine which part of the LPSs used was responsible for the observed reactions, Western blot preceded by LPS separation in polyacrylamide gel was performed. As can be seen in Fig. 1A, B, and C, all the antisera reacted with the low-molecular-mass species restricted to the core-lipid A region of all the LPSs.

Analyses of the reaction specificity observed in ELISA and Western blot are not enough to assess the similarity between the antigens. In this study this was

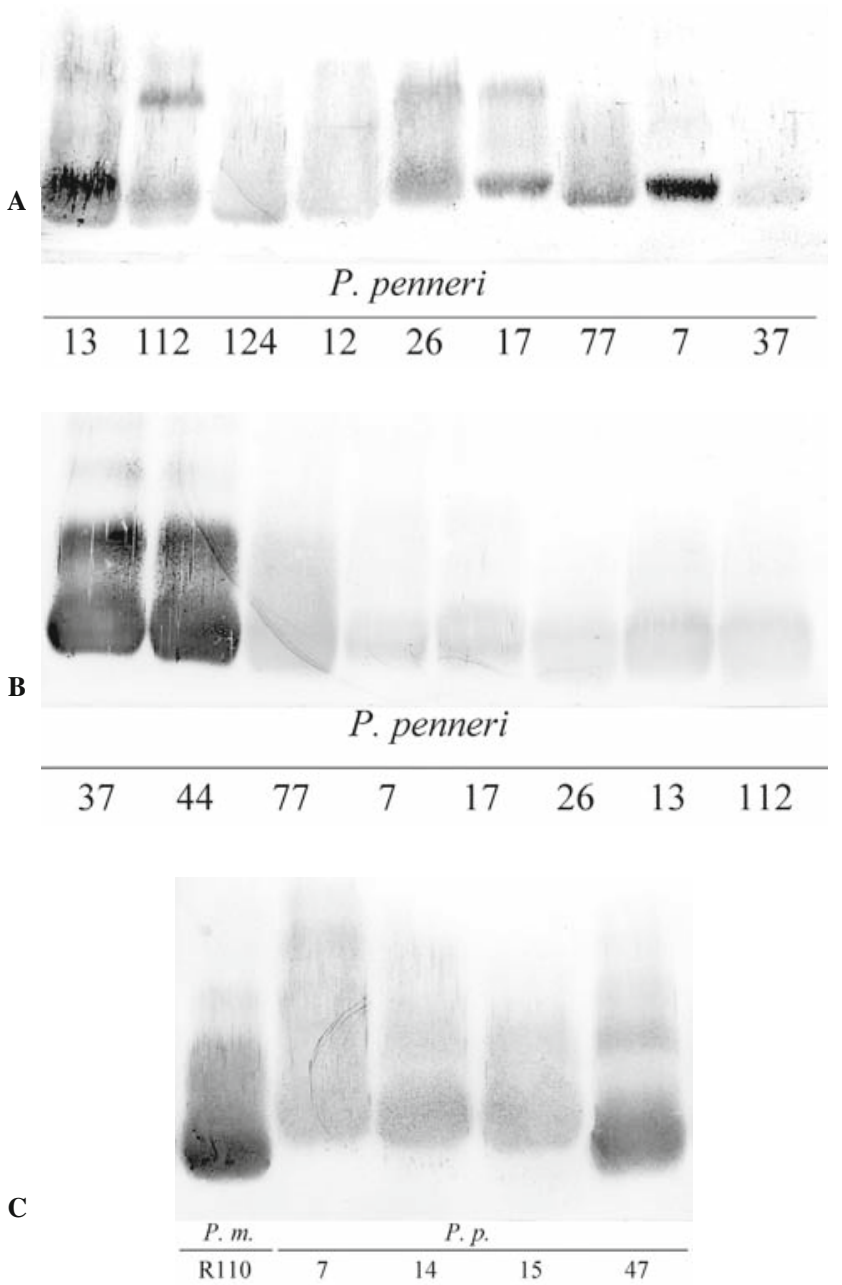

Fig. 1. Western blot of Proteus sp. LPSs with antisera against P. penneri 13 (A), P. penneri 37 (B), and P. mirabilis R110 (C).

done by the absorption of the tested antisera with SRBCs coated with the proper antigen. Each antiserum was absorbed with alkalized cross-reactive and homologous LPSs of one Proteus sp. strain and tested by PIH with all the antigens used for the absorption. The doses of the alkalized antigens needed for this assay had been selected before on the basis of results obtained for each antiserum with the method in question using increasing concentrations of each cross-reactive and homologous antigen (data not shown).

As can be seen in Table 2A, P. penneri 13 antiserum absorbed with $P$. penneri 13, 26, and 112 LPSs did not react with any of the antigens used, which suggests identity of the core regions of these three LPSs. On the other hand, the lower reaction of the non-absorbed antiserum (Table 2A, control) with P. penneri 26 LPS may indicate the presence of some structural differences between the core regions of $P$. penneri 26 LPS and the other two antigens (P. penneri 13 and 112). The second group of antigens reacting identically comprised two LPSs: P. penneri 12 and 124. Absorption of antiserum with P. penneri 12 and 124 LPSs completely removed anti- 
Table 2. Passive immunohemolysis data of the alkali-treated Proteus sp. LPSs with absorbed antisera against Proteus sp. strains ${ }^{\mathrm{a}, \mathrm{b}}$

\begin{tabular}{|c|c|c|c|c|c|c|c|c|c|}
\hline \multirow{2}{*}{$\begin{array}{l}\text { A. Alkali-treated LPS } \\
\text { used for absorption }\end{array}$} & \multicolumn{9}{|c|}{ Reciprocal titer of absorbed antiserum for alkali-treated LPS from $P$. penneri strains } \\
\hline & 13 & 112 & 12 & 124 & 26 & 17 & 7 & 37 & 77 \\
\hline \multicolumn{10}{|l|}{ P. penneri 13 antiserum } \\
\hline Control & 12,800 & 12,800 & 12,800 & 12,800 & 6,400 & 3,200 & 3,200 & 1,600 & 3,200 \\
\hline P. penneri 13 & - & - & - & - & - & - & - & - & - \\
\hline P. penneri 112 & - & - & - & - & - & - & - & - & - \\
\hline P. penneri 12 & 1,600 & 1,600 & - & - & 1,600 & 1,600 & 3,200 & 1,600 & 3,200 \\
\hline P. penneri 124 & 1,600 & 1,600 & - & - & 1,600 & 1,600 & 3,200 & 1,600 & 3,200 \\
\hline P. penneri 26 & - & - & - & - & - & - & - & - & - \\
\hline P. penneri 17 & 1,600 & 1,600 & 1,600 & 1,600 & - & - & - & - & - \\
\hline P. penneri 7 & 6,400 & 6,400 & 12,800 & 12,800 & 3,200 & 400 & - & - & - \\
\hline P. penneri 37 & 3,200 & 3,200 & 12,800 & 12,800 & 1,600 & 400 & - & - & - \\
\hline P. penneri 77 & 6,400 & 6,400 & 12,800 & 12,800 & 3,200 & 400 & - & - & - \\
\hline
\end{tabular}

B. Alkali-treated LPS Reciprocal titer of absorbed antiserum for alkali-treated LPS from P. penneri strains

\begin{tabular}{|c|c|c|c|c|c|c|c|c|}
\hline \multirow[b]{2}{*}{ used forabsorption } & & & & & & & & \\
\hline & 37 & 44 & 77 & 7 & 13 & 17 & 26 & 112 \\
\hline \multicolumn{9}{|l|}{ P. penneri 37 antiserum } \\
\hline Control & 51,200 & 51,200 & 6,400 & 1,600 & 1,600 & 1,600 & 1,600 & 1,600 \\
\hline P. penneri 37 & - & - & - & - & - & - & - & - \\
\hline P. penneri 44 & - & - & - & - & - & - & - & - \\
\hline P. penneri 77 & 3,200 & 3,200 & - & - & - & - & - & - \\
\hline P. penneri 7 & 25,600 & 25,600 & 1,600 & - & - & - & - & - \\
\hline P. penneri 13 & 25,600 & 25,600 & 1,600 & - & - & - & - & - \\
\hline P. penneri 17 & 25,600 & 25,600 & 1,600 & - & - & - & - & - \\
\hline P. penneri 26 & 25,600 & 25,600 & 1,600 & - & - & - & - & - \\
\hline P. penneri 112 & 25,600 & 25,600 & 1,600 & - & - & - & - & - \\
\hline \multirow{3}{*}{$\begin{array}{l}\text { C. Alkali-treated LPS } \\
\text { used for absorption }\end{array}$} & \multicolumn{8}{|c|}{ Reciprocal titer of absorbed antiserum for alkali-treated LPS from strains } \\
\hline & \multicolumn{3}{|c|}{ P. mirabilis } & \multicolumn{2}{|c|}{ P. penneri } & & & \\
\hline & & R110 & 7 & 14 & 15 & 47 & & \\
\hline \multicolumn{9}{|c|}{ P. mirabilis R110 antiserum } \\
\hline Control & & 51,200 & 800 & 800 & 1,600 & 25,600 & & \\
\hline P. mirabilis $\mathrm{R} 110$ & & - & - & - & - & - & & \\
\hline P. penneri 7 & & 25,600 & - & - & 400 & 12,800 & & \\
\hline P. penneri 14 & & 25,600 & - & - & 400 & 12,800 & & \\
\hline P. penneri 15 & & 12,800 & - & - & - & 6,400 & & \\
\hline P. penneri 47 & & - & - & - & - & - & & \\
\hline
\end{tabular}

${ }^{\mathrm{a}}$ Non-absorbed antisera were used as control; ${ }^{\mathrm{b}}$ lack of reaction.

bodies that recognized both antigens, decreased the titers of antiserum reactivity with $P$. penneri $13,17,26$, and 112 LPSs, and had no influence on the reactivity with $P$. penneri 7, 37, and 77 LPSs. These results suggest that probably identical core regions of $P$. penneri 12 and 124 LPSs have a common epitope with the $P$. penneri 13 , 17,26 , and 112 LPS core regions and that this fragment does not occur in the core regions of P. penneri 7, 37, and 77 LPSs. The last three antigens probably possess the other epitope in their core regions, also located in the core oligosaccharides of the remaining LPSs, except for P. penneri 12 and 124 . Therefore the absorption of antiserum with $P$. penneri 7, 37, and 77 LPSs influenced the reactions with them and $P$. penneri $13,17,26$, and 112 LPSs and did not bring about changes in the reactivity with $P$. penneri 12 and 124 LPSs.

The results from the absorption of $P$. penneri 37 antiserum confirm the outcomes of the previous assays. Like in ELISA and Western blot, in this assay three groups of antigens can also be distinguished (Table 2B): P. penneri 37 and 44, reacting identically and, when used for the absorption, abolishing the reactivity with all the antigens used; P. penneri 77 LPS, whose core region is probably very similar to the $P$. penneri 37 and 44 core oligosaccharides; and $P$. penneri 7, 13, 17, 26, and 112, all of 
which share the common epitope with $P$. penneri 37,44 , and 77 LPSs.

The absorption of the last antiserum, against mutant R110, with the homologous and P. penneri 47 LPS completely abolished its reactivity with all the antigens used (Table 2C). These results indicate that both LPSs may have identical core regions. The stronger reactivity of the non-absorbed antiserum (Tables 1, 2C - control) with the homologous LPS may result from the lack of O-polysaccharide chains as well as better accessibility of its core region for specific antibodies. The small differences which can be seen in the serological activities of P. penneri 7 and 14 LPSs, which reacted identically, and P. penneri 15 LPS may suggest a slightly different epitope specificity of these two groups of antigens.

Additionally, the antiserum directed against the $P$. penneri 44 rough mutant was used to confirm the identical serological specificity of the $P$. penneri 44 and 37 LPS core regions. This antiserum was tested with the two mentioned antigens using Western blot and PIH preceded by antiserum absorption with each antigen (data not shown). Both methods gave results identical to those obtained for $P$. penneri 37 antiserum (Fig. 1B, Table 2B). In Western blot the antiserum reacted strongly and identically with the slow migrating bands of both LPS preparations, and the absorption of the antiserum with the mentioned antigens removed all antibodies specific to them, which confirmed their core region identity.

The results of the serological studies performed using three tested antisera ( $P$. penneri 13 and 37 and $P$. mirabilis R110) were compared with the known chemical structures of the core region of homologous and cross-reactive LPSs in order to identify potential core epitopes determining the observed cross-reactions (Figs. 2, 3, 4). It is worth mentioning that the core region structures of some cross-reactive LPSs ( $P$. penneri 47, 77,112 , and 124) are still unknown. The epitope specificities of these LPSs can be putatively determined only by comparing them with the serological specificities of LPSs reacting identically to one of the four mentioned antigens and having a known structure of the core region.

As a result of these analyses, a few fragments of the Proteus LPS core region were suggested to act as epitopes. The first two, $\alpha$-GalN-( $1 \rightarrow 4)-\alpha-G a l A$ (epitope a) and $\alpha$-Hep-( $1 \rightarrow 2)$ - $\alpha$-DD-Hep (epitope $b$ ), were found in the core region of $P$. penneri 13 LPS (Fig. 2A). The former is responsible for the cross-reactions of $P$. penneri 13 antiserum with $P$. penneri 12, 17, and 26 LPSs and, probably, P. penneri 112 and 124 of unknown core region structure, and the latter accounts for the reactions with all the cross-reactive antigens except for P. penneri 12 (Fig. 2D) and P. penneri 124, which do not have this disaccharide in their core regions. Both epitopes were found, apart from the homologous LPS core region and, probably, the core region of the identically reacting $P$. penneri 112 LPS, also in the core oligosaccharides of $P$. penneri 17 and 26 LPSs (Figs. 2B, C). The former, despite having two epitopes in its core region, reacted more weakly than $P$. penneri 13,26 , and 112 LPSs. This was probably caused by the presence in the P. penneri 17 LPS core region of additional sugar components $(\beta-G a l-(1 \rightarrow 4)-(1 S)-$ GaloNAc) which are linked to epitope a and probably prevent this common fragment (epitope a) from being easily accessible to the specific antibodies (Fig. 2B). The other antigen possessing the sugar substituent linked to the GalN residue of epitope a is $P$. penneri 26 , which, in contrast to the $P$. penneri 17 core region, has only one sugar residue $(\beta-\mathrm{Glc})$ at this position (Fig. 2C). This fact and a non-stoichiometric amount (Vinogradov et al. 2002b) of this substituent are probably the reasons why the Glc residue makes the binding of antibodies by epitope a slightly more difficult. The detection in the tested antiserum of a higher number of antibodies specific to epitope a than to epitope $b$ means that the former is considered the main epitope. That is why absorption of the tested antiserum with $P$. penneri 12 and 124 LPSs, having only epitope a in their core regions, caused a more noticeable decrease in titer with $P$. penneri 13, 26, and 112 LPSs than after absorption with the LPSs of $P$. penneri 7, 37, and 77 , which possess epitope $\mathrm{b}$ only (Table $2 \mathrm{~A}$ ).

The $\alpha$-Hep- $(1 \rightarrow 2)-\alpha$-DD-Hep residue probably acts as epitope $\mathrm{b}$ in the cross-reactivity of $P$. penneri 37 antiserum with $P$. penneri $7,13,17,26,44,77$, and 112 LPSs, which have this disaccharide in their core regions (Fig. 3). Although this disaccharide is not the only fragment common to the core regions of these LPSs, it is likely to participate in these cross-reactions because its distal location may enable specific antibodies to access it more easily than the proximal part of the core region. What is more, there were no antiserum reactions observed in ELISA with P. penneri 12 and 124 LPSs without heptose disaccharide in their core regions (data not shown).

The results of all the serological assays showed activity of the $P$. penneri 77 core region similar to that obtained with $P$. penneri 37 and 44 LPSs. Although the structure of the $P$. penneri 77 core oligosaccharide has not been completely determined, some unpublished structural data obtained by other authors suggest that this core region, similar to the other cross-reactive LPSs, represents a third glycoform possessing the $\alpha$-Hep$-(1 \rightarrow 2)-\alpha-D D-H e p$ substituent. This confirms the results of the serological studies suggesting that heptose disaccharide is the epitope (b) contributing to the crossreaction of $P$. penneri 37 antiserum with $P$. penneri 77 LPS. That is why the absorption of the tested antiserum with this LPS influenced the reactivity with all the LPSs having the mentioned epitope in their core regions (Table 2B). Observing the stronger decrease in titer with $P$. penneri 37 and 44 LPSs after antiserum absorption with $P$. penneri 77 LPS than after absorption with $P$. penneri $7,13,17,26$, and 112 LPSs, it can be concluded that $P$. penneri 77 LPS has another core region fragment in common with $P$. penneri 37 and 44 antigens and that this epitope does not appear in the core regions of the 
A P. penneri $13\left(\mathrm{R}^{1}=P\right.$ Etn or $\mathrm{H} ; \mathrm{R}^{3}=\mathrm{H}$ or $\beta$-GalNA) (Vinogradov and Sidorczyk 2002)

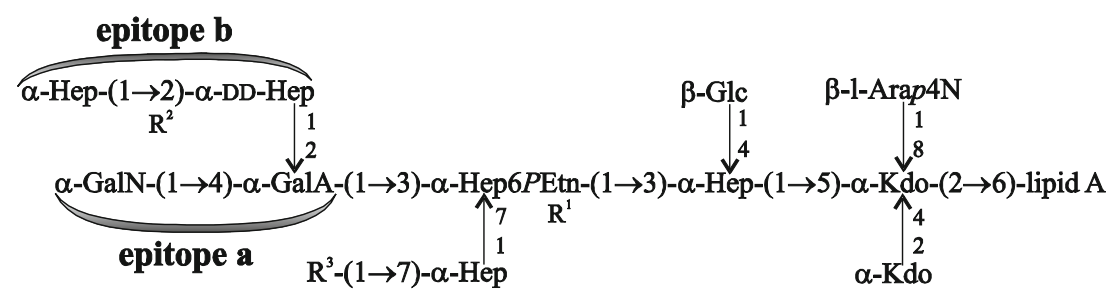

B P. Penneri $17\left(\mathrm{R}^{3}=\mathrm{H}\right)$ (Vinogradov et al. 2002b)

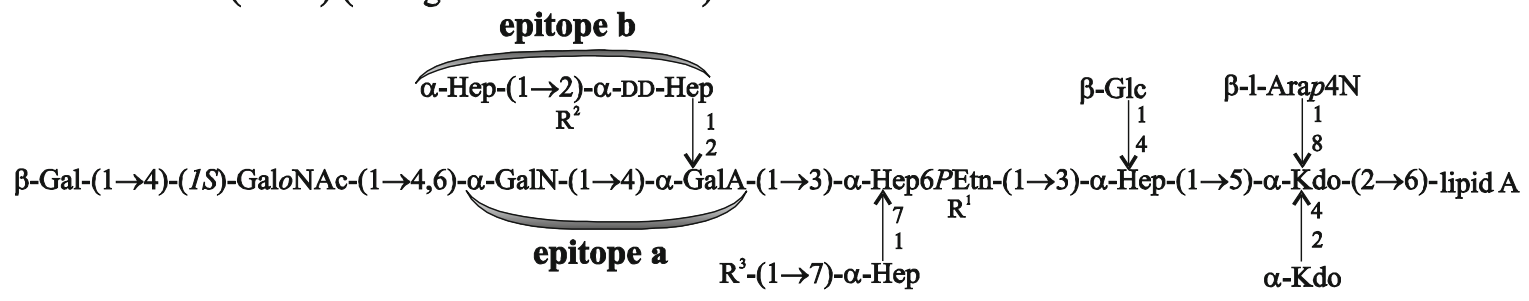

C P. penneri $26\left(\mathrm{R}^{3}=\mathrm{H}\right)$ (Vinogradov et al. 2002b)

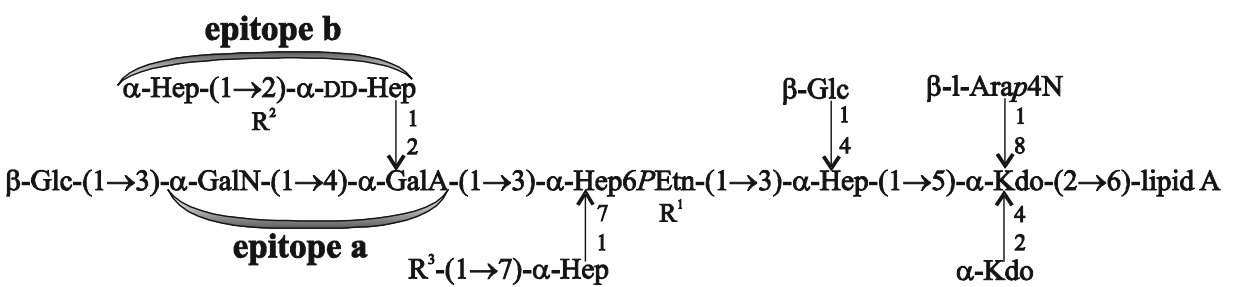

D P. penneri $12\left(\mathrm{R}^{3}=\mathrm{H}\right)($ Vinogradov and Sidorczyk 2002)

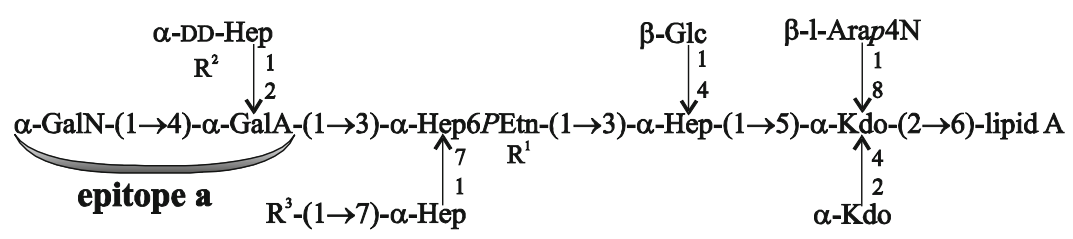

Fig. 2. Structures of the core-lipid A regions of $P$. penneri 13 LPS (A) and LPSs cross-reactive with $P$. penneri 13 antiserum (B, C, D). Abbreviations: Arap4N - 4-amino-4-deoxy-L-arabinopyranose, GalA - galacturonic acid, GalN - galactosamine, GalNA - amide of GalA with aliphatic polyamines, Hep - L-glycero-D-manno-heptose, DD-Hep - D-glycero-D-manno-heptose, Kdo - 3-deoxy-D-manno-oct-2-ulosonic acid.

other LPSs used. Comparing the core region structures of $P$. penneri 37 and 44 LPSs and the other LPSs, it was clearly seen that only the first two antigens possess a $\beta$ GlcNAc- $(1 \rightarrow 4)-\alpha-$ GlcN fragment in their core regions (Figs. 2A, B, C, 3, 4). This disaccharide may be the second epitope (c) involved in the cross-reaction with $P$. penneri 77 LPS. It should also be noted that the antibodies left in the antiserum after its absorption with P. penneri 77 LPS probably recognize another unidentified fragment of the P. penneri 37 and 44 LPSs core regions (Table 2B).

The epitope specificity of the LPS core region of the last tested strain, mutant R110, was determined previously by other authors (Bartodziejska et al. 1993). However, a reinvestigation of the structure of this mutant core region showed some structural differences between the present and the previous structures (Vinogradov et al. 2000). This encouraged us to describe the epitope specificity of the mutant R110 core region once again. Comparative analysis of the structur- al and serological data allowed identifying two fragments of the mutant R110 LPS core region reacting as potential epitopes (Fig. 4). The first one, $\alpha$-DD-Hep$-(1 \rightarrow 6)-\alpha-G l c N$ (epitope d), is suggested to be involved in the cross-reactivity with $P$. penneri 7,14 , and 15 LPSs and probably $P$. penneri 47 antigen, which may have the same core region structure as the homologous LPS. The other common epitope is associated with the trisaccharide $\alpha$-DD-Hep- $(1 \rightarrow 6)-\alpha$-GlcN- $(1 \rightarrow 4)-\alpha$-GalA (epitope e) in the core region of mutant R110 and P. penneri 15 LPSs and, probably, $P$. penneri 47 LPS. It is worth pointing out that this trisaccharide is also present in the $P$. penneri 7 and 14 LPS core regions, where it additionally involves a Gly residue separating the $\alpha$-GalA residue and $\alpha$-DD-Hep-( $1 \rightarrow 6)-\alpha-G l c N$ (epitope d). This amino acid probably prevents the specific antibodies from binding to the whole trisaccharide; therefore the antiserum absorption with $P$. penneri 7 and 14 LPSs (having the Gly residue in their core regions) left some 
P. penneri 37, $44\left(\mathrm{R}^{1}=P\right.$ Etn or $\mathrm{H} ; \mathrm{R}^{3}=\mathrm{H}$ or $\beta$-GalNA) (Vinogradov and Sidorczyk 2002)

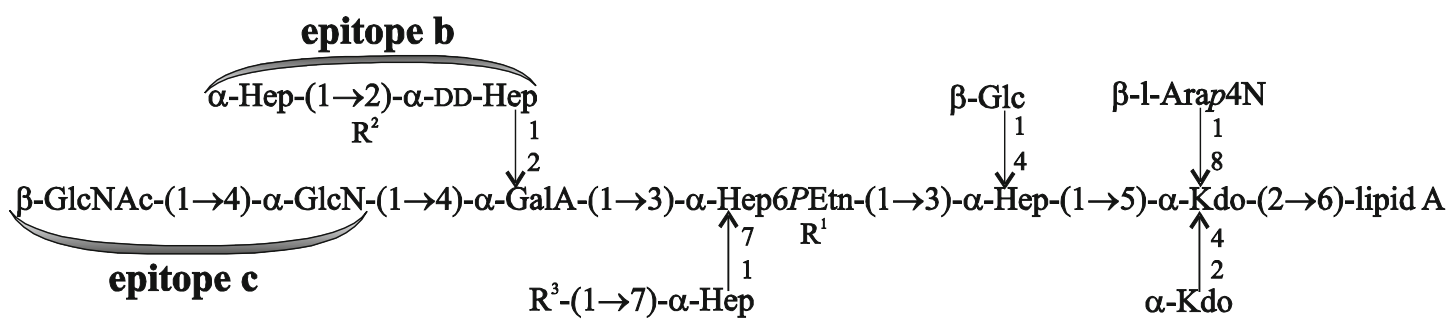

Fig. 3. Structures of the core-lipid A regions of $P$. penneri 37 and 44 LPSs.

small fraction of immunoglobulin recognizing epitope e in the $P$. penneri 15 core region (Table 2C).

The strong reactions of non-absorbed antiserum with the homologous and $P$. penneri 47 LPSs compared with the much weaker reactions observed with the other antigens (Tables 1, 2C, Fig. 1C) showed that some other unidentified core region fragments take part in the epitope specificity of mutant R110 LPS.

Summarizing, the tested antisera directed against the rough Proteus sp. strains reacted not only with the rough, but also with the smooth LPSs forms. In the observed cross-reactions the main role was played by fragments located in the most distal part of the LPS core region. Nevertheless, antibodies against some unidentified and probably more proximal fragments of LPS core regions have also been detected.

Acknowledgment: This work was supported by the Ministry of Science and Higher Education (Poland), grant no. N 401001 31/0009.

\section{REFERENCES}

Bartodziejska B, Radziejewska-Lebrecht J, Lipińska M et al (1993) Badanie swoistości epitopowej przeciwciał poliklonalnych przeciwko mutantom R Proteus mirabilis. Med Dośw Mikrobiol 45:99-102

Drzewiecka D, Zych K, Sidorczyk Z (2004) Characterization and serological classification of a collection of Proteus penneri clinical strains. Arch Immunol Ther Exp 52:121-128

Galanos C, Lüderitz O, Westphal O (1969) A new method for the extraction of $\mathrm{R}$ lipopolysaccharides. Eur $\mathrm{J}$ Biochem 9:245-249

Hickman FW, Steigerwalt AG, Farmer JJ III et al (1982) Identification of Proteus penneri sp. nov., formerly known as Proteus vulgaris indole negative or as Proteus vulgaris biogroup 1. J Clin Microbiol 15:1097-1102

Kołodziejska K, Perepelov AV, Zabłotni A et al (2006) Structure of the glycerol phosphate-containing O-polysaccharides and serological studies of the lipopolysaccharides of

P. mirabilis R110 (Vinogradov et al. 2000)

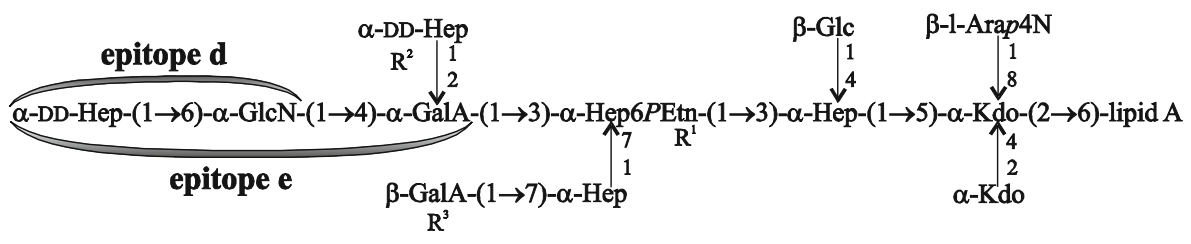

P. penneri $7\left(\mathrm{R}^{3}=\mathrm{H}\right)$, P. penneri $14\left(\mathrm{R}^{1}=P\right.$ Etn or $\mathrm{H} ; \mathrm{R}^{3}=\mathrm{H}$ or $\beta$-GalNA) (Vinogradov et al. 2002a)

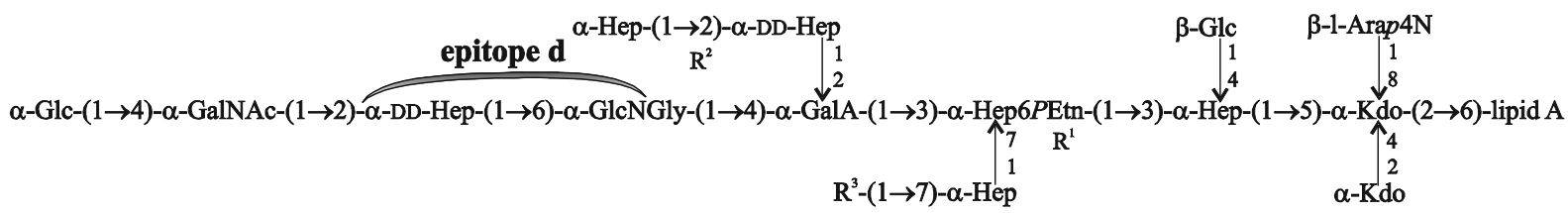

P. penneri $15\left(\mathrm{R}^{3}=\mathrm{H}\right)$ (Vinogradov et al. 2002a)

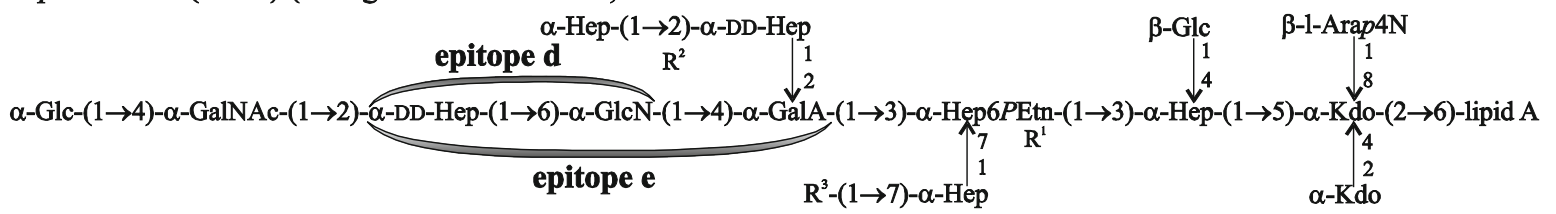

Fig. 4. Structures of the core-lipid A regions of mutant R110 LPS and the LPSs cross-reactive with P. mirabilis R110 antiserum. 
Proteus mirabilis CCUG 10704 (OE) and Proteus vulgaris TG 103 classified into a new Proteus serogroup, O54. FEMS Immunol Med Microbiol 47:267-274

Kondakova AN, Zych K, Senchenkova SN et al (2003) Structure of the O-polysaccharide of Proteus penneri 28 and Proteus vulgaris $\mathrm{O} 31$ and classification of P. penneri 26 and 28 in Proteus serogroup O31. FEMS Immunol Med Microbiol 39:87-93

Kotełko K, Gromska W, Papierz M et al (1977) Core region in Proteus mirabilis lipopolysaccharide. J Hyg Epidemiol Microbiol Immunol 21:271-284

Poxton IR (1995) Antibodies to lipopolysaccharide. J Immunol Methods 186:1-15

Sidorczyk Z, Toukach FV, Zych K (2002a) Structural and serological characterization of the lipopolysaccharide from Proteus penneri 20 and classification of the cross-reacting Proteus penneri strains 10, 16, 18, 20, 32 and 45 in Proteus serogroup O17. Arch Immunol Ther Exp 50:345-350

Sidorczyk Z, Toukach FV, Zych K et al (2002b) Structural and serological relatedness of the $\mathrm{O}$-antigens of Proteus penneri 1 and 4 from a novel Proteus serogroup O72. Eur J Biochem 269:358-363

Sidorczyk Z, Zych K, Toukach FV et al (2002c) Structure of the O-polysaccharide and classification of Proteus mirabilis strain G1 in Proteus serogroup O3. Eur J Biochem 269:1406-1412
Vinogradov E, Radziejewska-Lebrecht J, Kaca W (2000) The structure of the carbohydrate backbone of core-lipid A region of the lipopolysaccharides from Proteus mirabilis wild-type strain S1959 (serotype O3) and its Ra mutant R110/1959. Eur J Biochem 267:262-268

Vinogradov E, Sidorczyk Z (2002) The structure of the carbohydrate backbone of the rough type lipopolysaccharides from Proteus penneri strains 12, 13, 37 and 44. Carbohydr Res $337: 835-840$

Vinogradov E, Sidorczyk Z, Knirel YA (2002a) Structure of the core part of the lipopolysaccharides from Proteus penneri strains 7, 8, 14, 15, and 21. Carbohydr Res 337:643-649

Vinogradov E, Sidorczyk Z, Knirel YA (2002b) Structure of the lipopolysaccharide core region of the bacteria of the genus Proteus. Aust J Chem 55:61-67

Zych K, Kocharova NA, Kowalczyk M et al (2000) Structure of the O-specific polysaccharide of Proteus penneri 71 and classification of cross-reactive $P$. penneri strains to a new proposed serogroup O64. Eur J Biochem 267:808-814

Zych K, Perepelov AV, Baranowska A et al (2005) Structure and serological studies of the O-polysaccharide of Proteus penneri 75. Epitopes and subgroups of Proteus serogroup O73. FEMS Immunol Med Microbiol 43:141-148 